\title{
Overexpression of TIMP3 Protects Against Cardiac Ischemia/Reperfusion Injury by Inhibiting Myocardial Apoptosis Through ROS/Mapks Pathway
}

\author{
Hui Liua Xibo Jing ${ }^{\mathrm{b}}$ Aiqiao Dong ${ }^{\mathrm{a}}$ Baobao Bai ${ }^{\mathrm{a}}$ Haiyan Wang ${ }^{\mathrm{a}}$ \\ aDepartment of Cardiology, Tangdu Hospital, the Fourth Military University, Xi'an, ${ }^{b}$ Department of \\ Cardiology, the Second Affiliated Hospital of Shandong Traditional Chinese Medicine University, Jinan, \\ China
}

\section{Key Words}

Ischemia/reperfusion injury • Myocardial apoptosis $•$ TIMP3 $・$ ROS $•$ MAPKs

\begin{abstract}
Background/Aims: Myocardial ischemia/reperfusion (I/R) injury remains a great challenge in clinical therapy. Tissue inhibitor of metalloproteinases 3 (TIMP3) plays a crucial role in heart physiological and pathophysiological processes. However, the effects of TIMP3 on I/R injury remain unknown. Methods: C57BL/6 mice were infected with TIMP3 adenovirus by local delivery in myocardium followed by I/R operation or doxorubicin treatment. Neonatal rat cardiomyocytes were pretreated with TIMP3 adenovirus prior to anoxia/reoxygenation (A/R) treatment in vitro. Histology, echocardiography, in vivo phenotypical analysis, flow cytometry and western blotting were used to investigate the altered cardiac function and underlying mechanisms. Results: The results showed that upregulation of TIMP3 in myocardium markedly inhibited myocardial infarct areas and the cardiac dysfunction induced by I/R or by doxorubicin treatment. TUNEL staining revealed that TIMP3 overexpression attenuated I/R-induced myocardial apoptosis, accompanied by decreased Bax/Bcl-2 ratio, Cleaved Caspase-3 and Cleaved Caspase-9 expression. In vitro, A/R-induced cardiomyocyte apoptosis was abrogated by pharmacological inhibition of reactive oxygen species (ROS) production or MAPKs signaling. Attenuation of ROS production reversed A/R-induced MAPKs activation, whereas MAPKs inhibitors showed on effect on ROS production. Furthermore, in vivo or in vitro overexpression of TIMP3 significantly inhibited I/R- or A/R-induced ROS production and MAPKs activation. Conclusion: Our findings demonstrate that TIMP3 upregulation protects against cardiac I/R injury through inhibiting myocardial apoptosis. The mechanism may be related to inhibition of ROS-initiated MAPKs pathway. This study suggests that TIMP3 may be a potential therapeutic target for the treatment of I/R injury.
\end{abstract}




\section{Cellular Physiology Cell Physiol Biochem 2017;44:1011-1023 \begin{tabular}{l|l|l} 
and Biochemistry & DOI: 10.1159/000485401 & $\begin{array}{l}\text { C } 2017 \text { The Author(s). Published by S. Karger AG, Basel } \\
\text { www.karger.com/cpb }\end{array}$
\end{tabular}}

Liu et al.: the Cardiac Protective Effect of TIMP3

\section{Introduction}

Myocardial ischemia caused by coronary blockage underlies the majority of cardiovascular-related deaths $[1,2]$. It is clinically presented as permanent and fierce retrosternal pain, resulting in myocardial infarction, shock, cardiac arrhythmia or heart failure $[3,4]$. Early restoration of blood flow into the ischemia area is the most commonly therapeutic strategy, such as coronary angioplasty, percutaneous coronary intervention and coronary artery bypass grafting, which can save ischemic myocardium and rescue the patient life [1]. However, ischemia/reperfusion (I/R) inevitably leads to acute tissue injury, seriously limiting the clinical curative effect [5]. Hence, understanding the underlying mechanisms and developing novel strategies are important for the prevention and treatment of cardiac I/R injury.

Since fibrosis is the major cause of myocardial infarction and heart failure, the regulation of extracellular matrix (ECM) deposition has been received increasing attentions [6]. The turnover of ECM is mediated by a family of enzymes, such as matrix metalloproteinases (MMPs), disintegrins and metalloproteinases (ADAMs) [7, 8]. The tissue inhibitor of metalloproteinases (TIMPs) plays a critical role in regulating MMPs activity and ECM formation, because the imbalance between MMPs and TIMPs markedly disrupts ECM integrity in multiple cardiovascular diseases $[9,10]$. Among the four TIMPs, TIMP3 is a unique member, which not only inhibits MMPs activity, but also functions as a potent inhibitor of ADAMs $[11,12]$, indicating a crucial role of TIMP3 in the regulation of ECM deposition.

Accumulating evidences have demonstrated the important role of TIMP3 in cardiovascular disorders. ApoE $\%$ TIMP3 $\%$ mice showed accelerated atherosclerosis and aneurysms, while upregualtion of TIMP3 could limit the development of atherosclerosis $[10,13,14]$. Loss of TIMP3 exacerbated sepsis- or hypertension-induced endothelial cell barrier dysfunction $[11,15]$. Correspondingly, there are also some limited studies indicating TIMP3 has a role in regulation of heart function. Mouse heart lacking TIMP3 amplified the cross-talk between TGF $\beta 1$ and TNF signaling with an early onset of cardiac response to pressure overload, which in turn developed marked myocardial fibrosis [16]. In addition, TIMP3 deficiency accelerated ADAMs and MMPs-mediated dilated and hypertrophic cardiomyopathies $[17,18]$. However, the role of TIMP3 in the context of myocardial fibrosis and infarction is relatively less investigated when considering classical risk factor such as I/R injury. Thus, this study aims to investigate whether TIMP3 prevents against cardiac I/R injury and to explore the underlying mechanisms. Our work provides a novel understanding of TIMP3 in the pathogenesis of myocardial dysfunction and further highlights the potential therapeutic functions of TIMP3.

\section{Materials and Methods}

\section{Materials and reagents}

Pentobarbital sodium, doxorubicin (Dox), Evans Blue dye, 2, 3,5-triphenyltetrazolium chloride (TTC), DAPI, Tempol, NAC, SB203580, SP600125, and PD98059 were obtained from Sigma-Aldrich (MO, USA). Mouse TIMP3 adenovirus (Ad-TIMP3), rat Ad-TIMP3, and their corresponding negative controls (Ad-Lacz) were purchased from Sunbio Medical Biotechnology (Shanghai, China). Dulbecco's Modified Eagle's Medium (DMEM), fetal bovine serum (FBS), streptomycin, penicillin and $\mathrm{H}_{2}$ DCF-DA were from Invitrogen (CA, USA)

\section{$I / R$ injury model}

Male C57BL/ 6 mice (8 weeks old) were obtained from the Model Animal Research Center of Nanjing University (MARC, Nanjing, China). All animal experiments were performed according to the guidelines of the Institutional Animal Care and Use Committee of the Fourth Military University. In the current study, Ad-TIMP3 was used to upregulate the cardiac endogenous TIMP3 expression in vivo using a local delivery method as previously described [9]. 3 days before I/R operation or Dox treatment, mice were anesthetized with pentobarbital sodium (50mg/kg, i.p.) and the chest was opened. A total of $2 \times 10^{9}$ pfu of Ad-Lacz or $2 \times$ 
$10^{9} \mathrm{pfu}$ of Ad-TIMP3 in a final volume of $50 \mu \mathrm{l}$ was injected into the left ventricular (LV) cavity through the tip of the heart with a 36-gauge needle. Intramuscular injections were made in approximately 10 different sites. After adenovirus delivery, the chest was closed with sutures and the mice were transferred back to the cages for recovery. To perform I/R surgery, after anesthesia, the animals were ventilated on a rodent respirator via a tracheostomy. A later thoracotomy was performed, and a reversible snare occlude was placed around the left anterior descending coronary artery at 2 to $3 \mathrm{~mm}$ from the tip of the left auricle. I/R injury was induced by tightening the snare for $30 \mathrm{~min}$, followed by reperfusion for $4 \mathrm{~h}$ (acute injury) or for 8 weeks (chronic injury). Sham-operated mice underwent the same procedure except the snare was left untied.

Similar to I/R injury, a well-established mouse model of chronic Dox infusion was used to further investigate the role of TIMP3 in regulating cardiotoxicity and heart failure. After adenovirus delivery as described above, mice were anesthetized and implanted with a Mini osmotic pump (Alzet pumps Model 2002, Alzet, CA, USA), which was loaded with $200 \mu$ of either saline (vehicle for Dox), or Dox at a dose of 15 $\mathrm{mg} / \mathrm{kg}$ of body weight, at a rate of $0.5 \mu \mathrm{L} / \mathrm{h}$, for a period of 14 days.

\section{Echocardiography}

Cardiac function of mice was evaluated by echocardiographic analysis using an animal specific instrument (Vevo770; VisualSonics, ON, Canada) as previously described [5, 19]. During I/R operation, ischemia was confirmed by observing ST segment elevation and QRS widen on echocardiograms. At the end of animal experiments, LV ejection fraction (EF) and percentage fractional shortening (FS) were calculated.

\section{Infarct size measurement}

Myocardium infarct size was measured as previously described [19, 20]. After anesthesia, the heart was excised, and $1 \mathrm{~mL}$ of $5 \%$ Evans Blue dye was injected into the ascending aorta to visualize the nonischemic area. The coronary artery was re-ligated at the site of occlusion prior to Evans blue infusion. Then, the ventricular tissues were kept overnight at $-80^{\circ} \mathrm{C}$ and sliced into $2 \mathrm{~mm}$ thick sections, which were incubated with $1 \%$ TTC for $15 \mathrm{~min}$ at $37^{\circ} \mathrm{C}$ to visualize the ischemic but not infarct area (area at risk, AAR). The unstained region manifested infarcted myocardium. Infarct area and AAR were measured with computer assisted planimetry (Image 1.57, NIH, MD, USA). Infarct size was expressed as infarct area divided by AAR (IF/AAR).

\section{Assay of lactate dehydrogenase (LDH) release}

At the end of the experiments, blood samples were immediately collected from the abdominal vena cava and centrifuged for $10 \mathrm{~min}$ at $3000 \times \mathrm{g}$ to obtained serum. LDH release was spectrophotometrically measured using the CytoTox 96 non-Radioactive Cytotoxicity Assay kit (Promega, G1780, WI, USA) according to the manufacturer's instructions.

\section{Masson trichrome staining}

Slides were incubated with Masson trichrome reagent for $15 \mathrm{~min}$, followed by counterstaining with hematoxylin. To measure the extent of the increase of cardiac fibrosis quantitatively, the collagen tissue area was determined using quantitative image analysis software (Image 1.57) by rating the area occupied by Masson trichrome-positive area and expressed as percentage of the full LV area.

\section{Cardiomyocytes isolation and treatment}

Rat ventricular cardiomyocytes for in vitro experiments were isolated from 1-to 2-day-old neonatal Sprague-Dawley rats as described previously [21]. Cells were maintained in DMEM supplemented with $10 \% \mathrm{FBS}$ and antibiotics (streptomycin/penicillin, $100 \mu \mathrm{g} / \mathrm{mL}$ ) in a humidified incubator with $5 \% \mathrm{CO}_{2}$ at $37^{\circ} \mathrm{C}$. Cardiomyocytes anoxia/reoxygenation (A/R) model was performed as previously described [22]. To induce cell anoxia, cardiomyocytes were cultured in serum-free DMEM saturated with $95 \% \mathrm{~N}_{2} / 5 \% \mathrm{CO}_{2}$ in an anaerobic chamber with $95 \% \mathrm{~N}_{2} / 5 \% \mathrm{CO}_{2}$ for $24 \mathrm{~h}$. For reoxygenation, the culture medium was changed to DMEM/10\% FBS, and cells were incubated in a $5 \% \mathrm{CO}_{2} / 37^{\circ} \mathrm{C}$ incubator for $24 \mathrm{~h}$. Adenovirus carrying TIMP3 was added to upregulate TIMP3 expression in cultured cells for $24 \mathrm{~h}$ before A/R treatment.

\section{TUNEL staining}

Apoptosis in myocardial tissues or cultured cardiomyocytes was analyzed by performing a TUNEL assay suing an In situ Cell Death Detection kit (Cat. No. 11684817910, Roche, Mannheim, Germany). 


\section{Cellular Physiology Cell Physiol Biochem 2017;44:1011-1023 \begin{tabular}{l|l|l} 
and Biochemistry Published online: November 27, 2017 & $\begin{array}{l}\text { (c) } 2017 \text { The Author(s). Published by S. Karger AG, Basel } \\
\text { www.karger.com/cpb }\end{array}$
\end{tabular}}

Liu et al.: the Cardiac Protective Effect of TIMP3

For measurement of apoptosis in vivo, the frozen heart sections were incubated with TUNEL Reaction Mixture at $37{ }^{\circ} \mathrm{C}$ for $60 \mathrm{~min}$. For evaluation cardiomyocyte apoptosis in vitro, cells were fixed with $4 \%$ paraformaldehyde, permeated with $0.1 \%$ Triton X-100, and incubated with TUNEL Reaction Mixture for 60 $\min$ at $37^{\circ} \mathrm{C}$. After a $5 \mathrm{~min}$ DAPI counterstain at room temperature, cells were photographed with a confocal microscope (FV1000, Olympus, Tokoyo, Japan). The percentage of TUNEL-positive cells was calculated by ratio of (stained apoptotic cells)/(total number of cells) $\times 100 \%$.

\section{Western blotting}

Protein was extracted from cardiomyocytes or from the anterior wall of LV using RIPA lysis buffer (Beyotime Institute of Biotechnology, Shanghai, China) with protease inhibitor cocktail (Thermo, MA, USA). Protein concentration was determined using a Bradford assay (Bio-Rad Laboratories, CA, USA). The extracted protein $(60 \mu \mathrm{g})$ was separated by SDS-PAGE gel and then transferred onto a Polyvinylidene Fluoride (PVDF) membrane (Millipore, MA, USA). After blocking, the membranes were respectively probed with the following specific antibodies: p-p38 (1:1000, sc-7973), p38 (1:1000, sc-7149), p-JNK (1:500, sc-6254), JNK (1:1000, sc-7345), p-Erk1/2 (1:500, sc-136521), Erk1/2 (1:2000, sc-135900) and $\beta$-actin (1:2000, sc-81178) (Santa Cruz, CA, USA); Bcl-2 (1:1000, \#3498), Caspase-9 (1:1000, \#9508) and Caspase-3 (1:500, \#9662) (Cell signaling Technology, MA, USA). The horseradish peroxidase-conjugated secondary antibodies were purchased from Beyotime. Bands were visualized by an enhanced chemiluminescence system (Thermo Scientific, Pittsburgh, USA) and quantified by Image 1.57 software.

Reactive oxygen species (ROS) detection

Heart sections and cardiomyocytes were incubated with $\mathrm{H}_{2} \mathrm{DCF}-\mathrm{DA}(10 \mu \mathrm{mol} / \mathrm{L})$ for $30 \mathrm{~min}$ at $37^{\circ} \mathrm{C}$ in dark and then rinsed with PBS three times. The fluorescence was observed with a fluorescence microscope (BX61, Olympus) and the fluorescence intensity was analyzed by Image 1.57 software.

\section{Cell apoptosis assay}

Cardiomyocyte apoptosis was determined by a FITC-Annexin V Apoptosis Detection Kit (Beyotime, C1063) using flow cytometry. Cells were digested with trypsin, harvested by centrifugation, and then resuspended in a binding buffer of Annexin V-FITC and propidium iodide (PI) for $15 \mathrm{~min}$ at room temperature in dark. The apoptotic cells were counted by Accuri C6 flow cytometry (BD Biosciences, CA, USA) and the apoptosis percentage was calculated with the accompanying software (CFlow Plus software, BD Biosciences).

\section{Statistical analysis}

The results were expressed as mean value \pm standard error of mean (SEM). $n$ represents the number of experiments. Statistical analysis of data was performed by one-way ANOVA, followed by the Bonferroni multiple comparison test. $\mathrm{P}<0.05$ was considered statistically significant.

\section{Results}

Overexpression of TIMP3 diminishes I/R-induced myocardial injury

Myocardial infarction was measured by Evans blue-TTC staining after $4 \mathrm{~h}$ of reperfusion. The blue color denotes the non-ischemic area, while the pink and white regions represent as the ischemic and infarct area, respectively. As shown in Fig. 1A, no infarct area was found in myocardium from sham-operated mice. However, I/R mice exhibited apparent infarction. Compared with I/R mice, overexpression of TIMP3 with adenoviral vector encoding mouse TIMP3 (Ad-TIMP3) significantly decreased the myocardial infarct area. This was supported by the quantification of IF/AAR (Fig. 1B). LDH is a well-known indicator of cytotoxicity during various myocardial injuries, including I/R. The results showed that the release of $\mathrm{LDH}$ in I/R mice was higher than that of sham mice, and Ad-TIMP3 administration lowered this kind of increase (Fig. 1C). To further explore the potential contribution of TIMP3 in the sequelae of I/R injury, a chronic I/R experimental model was induced by 30 min ischemia and 8 weeks reperfusion. In this model, TIMP3 overexpression resulted in a reduction in myocardial fibrosis and the ratio of heart weight to body weight (Fig. 1D-F). Moreover, 


\section{Cellular Physiology and Biochemistry}

Fig. 1. TIMP3 overexpression protects against I/R-induced myocardial injury and heart failure. (A) Representative photographs of myocardial tissue sections from sham, and I/R (30 min ischemia and $4 \mathrm{~h}$ reperfusion) mice treated with Ad-Lacz or Ad-TIMP3. (B) Myocardial infarct size expressed as ratio of infarct size (IF) to area at risk (AAR). $n=8$ in each group. (C) Serum LDH concentration was examined. $n=15$ in each group. (D) Representative images revealed Masson trichrome staining in the hearts isolated from sham, I/R (30 min ischemia and 8 weeks reperfusion), and I/R mice treated with Ad-Lacz or AdTIMP3. (E) The degree of myocardial fibrosis was determined by computer-based morphometric analysis. (F) The ratio of heart to body weight was measured. (G

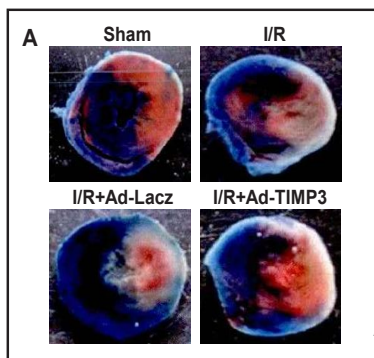

D

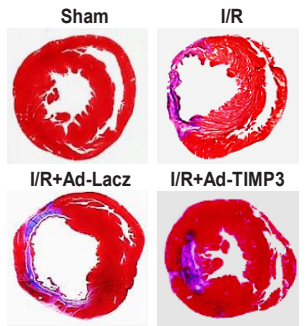

G

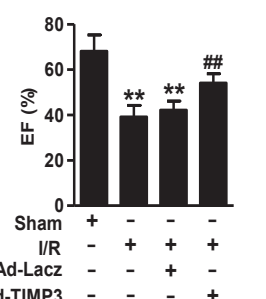

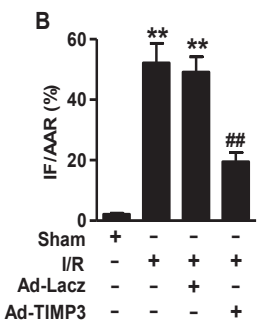
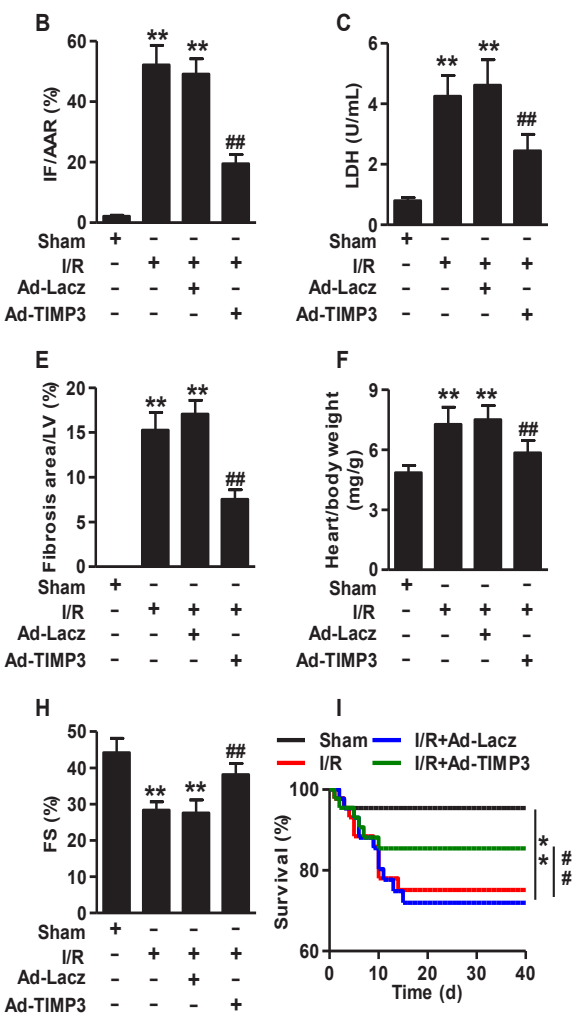
and $\mathrm{H}$ ) Echocardiographic analy-

sis ejection fraction (EF, G), fractional shortening (FS, H). n=14 in each group. (I) Mice survival curves were monitored until day 40 after the beginning of I/R operation. $n=26$ in each group. Data were expressed as mean value \pm SEM. ${ }^{* *} \mathrm{P}<0.01$ vs. sham; \#\#P<0.01 vs. I/R.

echocardiography results showed that I/R-induced the impairment of cardiac function, as evidenced by decreased EF and FS, was significantly ameliorated in Ad-TIMP3-treated mice (Fig. 1G-H). In addition, TIMP3 upregulation markedly lowered chronic I/R-induced mortality (Fig. 1I). These data suggest that TIMP3 is important for I/R-induced myocardial injury and its associated sequelae.

\section{TIMP3 upregulation restrains Dox-induced myocardial injury}

Dox, an anthracycline antibiotic, is widely used for the treatment of a variety of cancers. However, its clinical utility is largely limited by the development of severe cardiotoxicity. To investigate whether TIMP3 ameliorates myocardial injury caused by different insults, we determined the contribution of TIMP3 in Dox-induced myocardial dysfunction and heart failure. As expected, LDH release was significantly elevated after Dox insult. Overexpression of TIMP3 reduced its levels as compared with mice treated with Dox alone (Fig. 2A). Moreover, Dox challenge induced obvious cardiac remodeling, including decreased fibrosis and hypertrophy, which were remarkably ameliorated after Ad-TIMP3 administration (Fig. 2B-D). Echocardiography tracing showed EF and FS were more severely reduced in the Dox alone-treated mice than in that mice co-treated with Dox and Ad-TIMP3 (Fig. 2E and F). Furthermore, Ad-TIMP3-treated mice also markedly decreased the susceptibility to Dox-induced mortality (Fig. 2G). The results indicate that TIMP3 is indispensable for Doxinduced cardiotoxicity.

TIMP3 attenuates myocardial apoptosis induced by I/R injury

To elucidate the possible mechanism of TIMP3-mediated cardiac protection in vivo, TUNEL staining was applied to assess the role of TIMP3 in myocardial apoptosis. The 


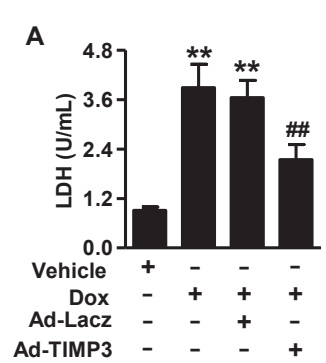

D

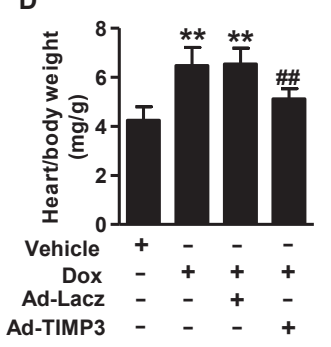

B

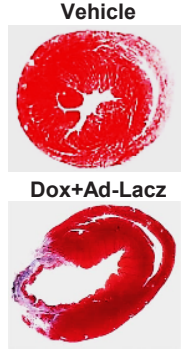

E

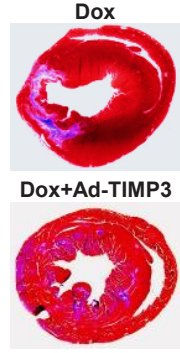

$\mathbf{F}$

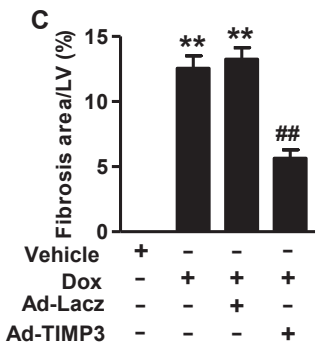

G

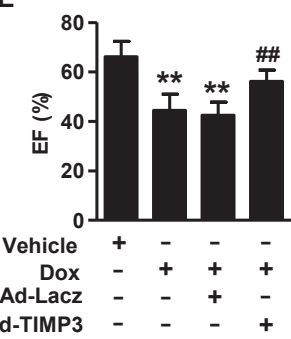

$\mathbf{F}$
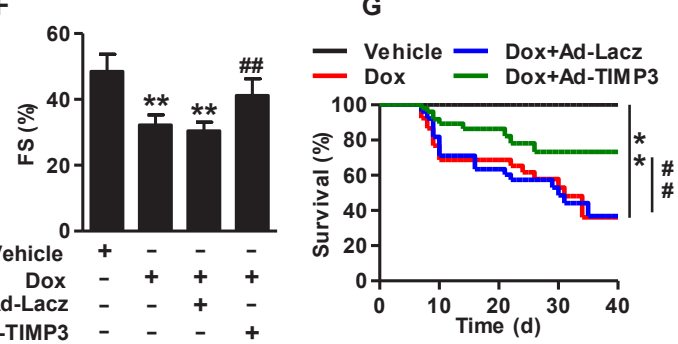

Fig. 2. TIMP3 upregulation alleviates Dox-induced myocardial dysfunction. (A) Mice were pretreated with Ad-Lacz or Ad-TIMP3, and then were infused with Dox (15 mg/kg of body weight) with an osmotic pump for 14 days. Serum LDH concentration was examined. $n=13$ in each group. (B) Representative images revealed Masson trichrome staining in the hearts isolated from vehicle, Dox, and Dox mice treated with Ad-Lacz or Ad-TIMP3. (C) The degree of myocardial fibrosis was determined by computer-based morphometric analysis. (D) Quantitative data of the heart-to-body weight ratio. (E and F) Echocardiographic analysis ejection fraction (EF, E), fractional shortening (FS, F). $n=11$ in each group. (G) Survival curves were recorded until day 40 after the start of Dox treatment. $\mathrm{n}=24$ in each group. ${ }^{* *} \mathrm{P}<0.01$ vs. vehicle; $\# \# \mathrm{P}<0.01$ vs. Dox.

Fig. 3. TIMP3 ameliorates I/Rinduced cardiomyocyte apoptosis. (A) Apoptosis was detected by TUNEL staining. Representative TUNEL and $\alpha$-actinin stained photographs of cardiomyocytes in heart sections. TUNEL staining was green colour representing apoptotic cells; nuclei stained by DAPI showed blue; cardiomyocytes were labeled with $\alpha$-actinin showing red colour. (B) Quantification of apoptotic cardiomyocytes in four groups. (C) The protein expressions of Bcl-2, Bax, Cleaved Caspase- 9 and Cleaved Caspase- 3 were determined using western blotting analysis. (D) Densitometric analysis of the above proteins expressions. $\mathrm{n}=7$ in each group. ${ }^{* *} \mathrm{P}<0.01$ vs. sham; \#\# $\mathrm{P}<0.01$ vs.

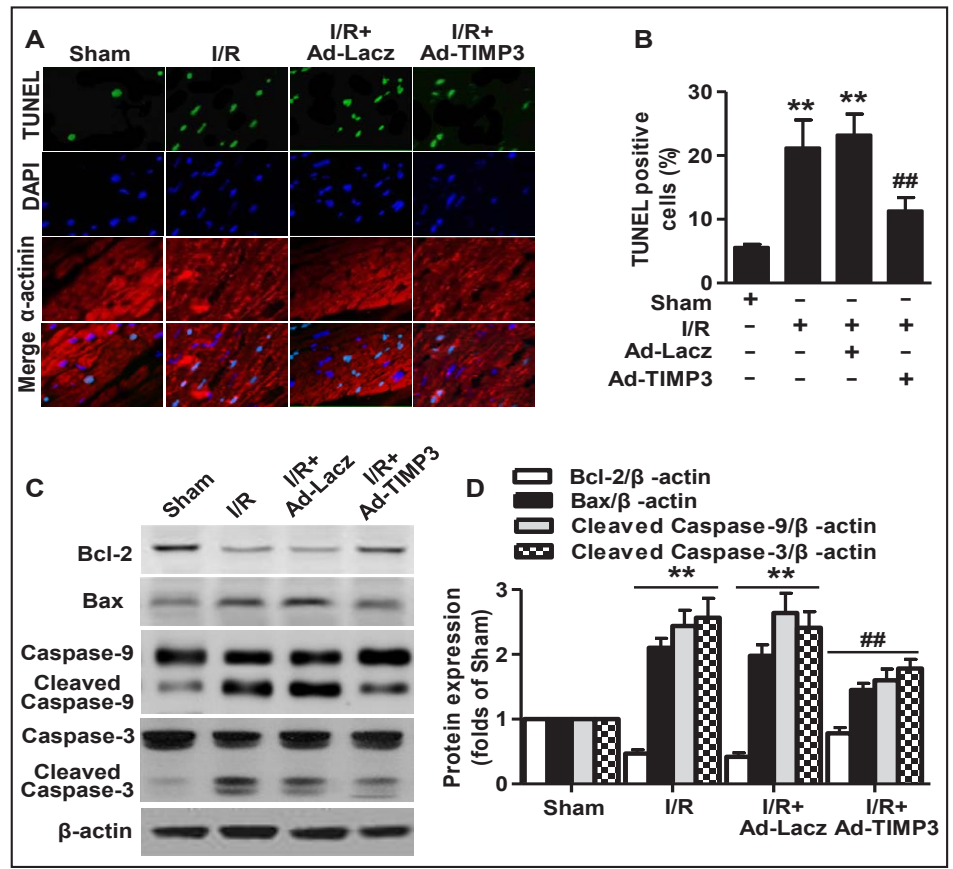
I/R.

cardiomyocytes and nucleus were identified by immunofluorescence with $\alpha$-actinin antibody and DAPI staining, respectively. As shown in Fig. 3A, there were little TUNEL-positive cells 
Fig. 4. ROS and MAPKs signaling pathways are involved in cardiomyocyte apoptosis induced by I/R. (A) Representative images for heart sections from sham or I/R mice loaded with $\mathrm{H}_{2}$ DCF-DA (10 $\mu \mathrm{mol} / \mathrm{L})$ was captured with a fluorescence microscopy. (B) Quantitative analysis of DCF fluorescence intensity. (C) Western blotting analysis of p38, JNK and Erk1/2 phosphorylation. (D) Densitometric analysis of the phosphorylated levels of p38, JNK and Erk1/2. $\mathrm{n}=7$ in each group.
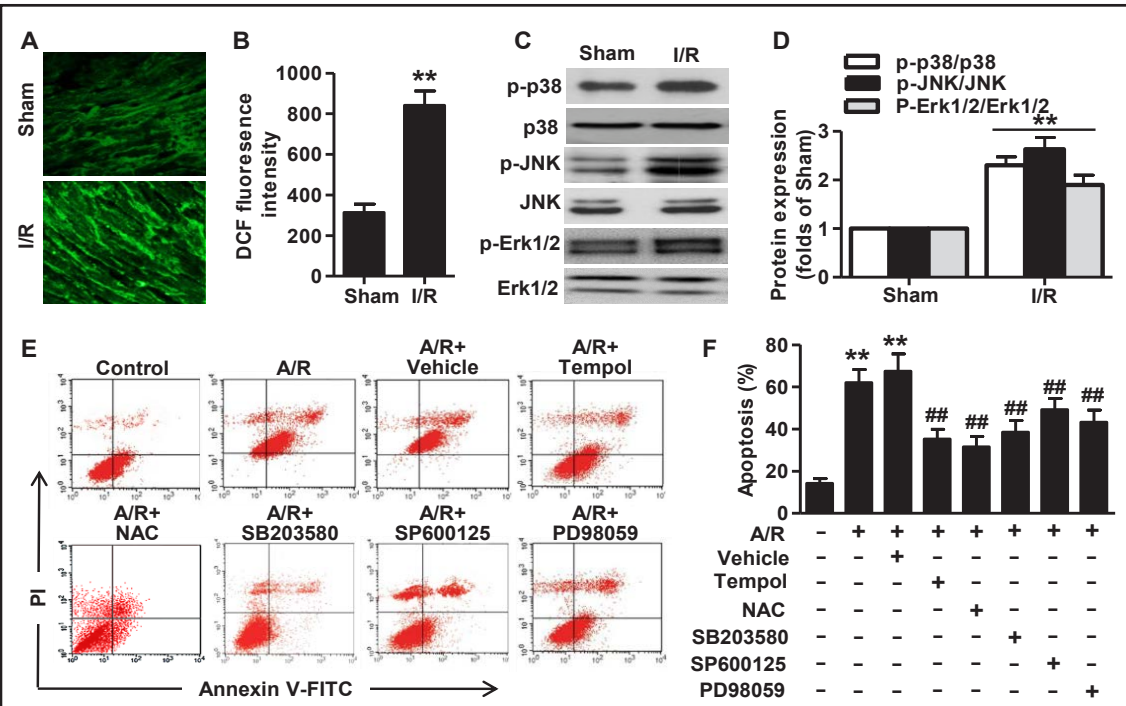

G
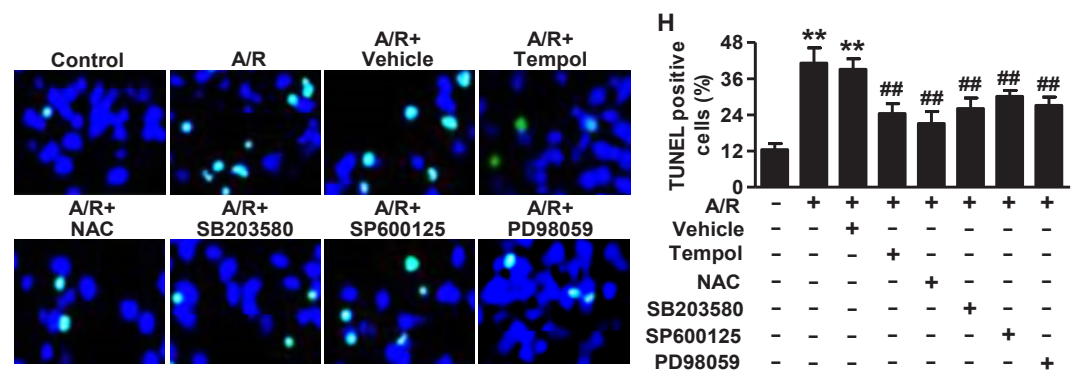

$* * \mathrm{P}<0.01$ vs. sham.

(E) Rat ventricular cardiomyocytes were pretreated with Tempol (1 mmol/L), NAC (5 mmol/L), SB203580 $(1 \mu \mathrm{mol} / \mathrm{L})$, SP600125 (10 $\mu \mathrm{mol} / \mathrm{L})$ or PD98059 (10 $\mu \mathrm{mol} / \mathrm{L})$ for $30 \mathrm{~min}$, followed by A/R treatment for 48 h. Cell apoptosis was determined by Annexin V/PI staining using flow cytometry. (F) Quantitative analysis of the percentage of apoptotic cells. (G) TUNEL (green) and DAPI staining (blue) of cardiomyocytes following treatment as mentioned above. $(\mathrm{H})$ The percentage of TUNEL positive cells was calculated. ${ }^{* *} \mathrm{P}<0.01 \mathrm{vs}$. control; \#\#P<0.01 vs. A/R, $\mathrm{n}=6$.

in hearts from sham mice. After $30 \mathrm{~min}$ ischemia and $4 \mathrm{~h}$ reperfusion, the percentage of TUNEL-positive cells was significantly increased. However, the apoptotic cells in I/R mice was markedly reduced by TIMP3 overexpression, whereas Ad-Lacz had no obvious effect on I/R-induced myocardial apoptosis (Fig. 3A). The statistical result of the percentage of TUNEL-positive cells validated the alteration in myocardium apoptosis (Fig. 3B). To further investigate how TIMP3 protects against I/R-induced myocardial apoptosis, we determined apoptosis-related molecules expressions using western blotting assay. The results showed that Bcl-2 expression was significantly decreased, whereas Bax, Cleaved Caspase- 9 and Cleaved Caspase- 3 expressions were increased, at $4 \mathrm{~h}$ after reperfusion. Upregulation of TIMP3 prevents I/R-induced inhibition of Bcl-2, and it also blocked the promotion of Bax, Cleaved Caspase-3 and Cleaved Caspase-9 (Fig. 3C and D). These data suggest that TIMP3 protects against myocardial injury, probably through inhibition of myocardial apoptosis.

\section{Activation of ROS and MAPKs signaling pathways in I/R-induced myocardial apoptosis}

Since both ROS and MAPKs have been considered important mediators of cell apoptosis [23-25], we initially investigated the effect I/R injury on these pathways activation in cardiomyocytes. $\mathrm{H}_{2}$ DCF-DA staining showed that the ROS production in heart slices from I/R mice was dramatically increased as compared with sham mice (Fig. 4A and B). In addition, 


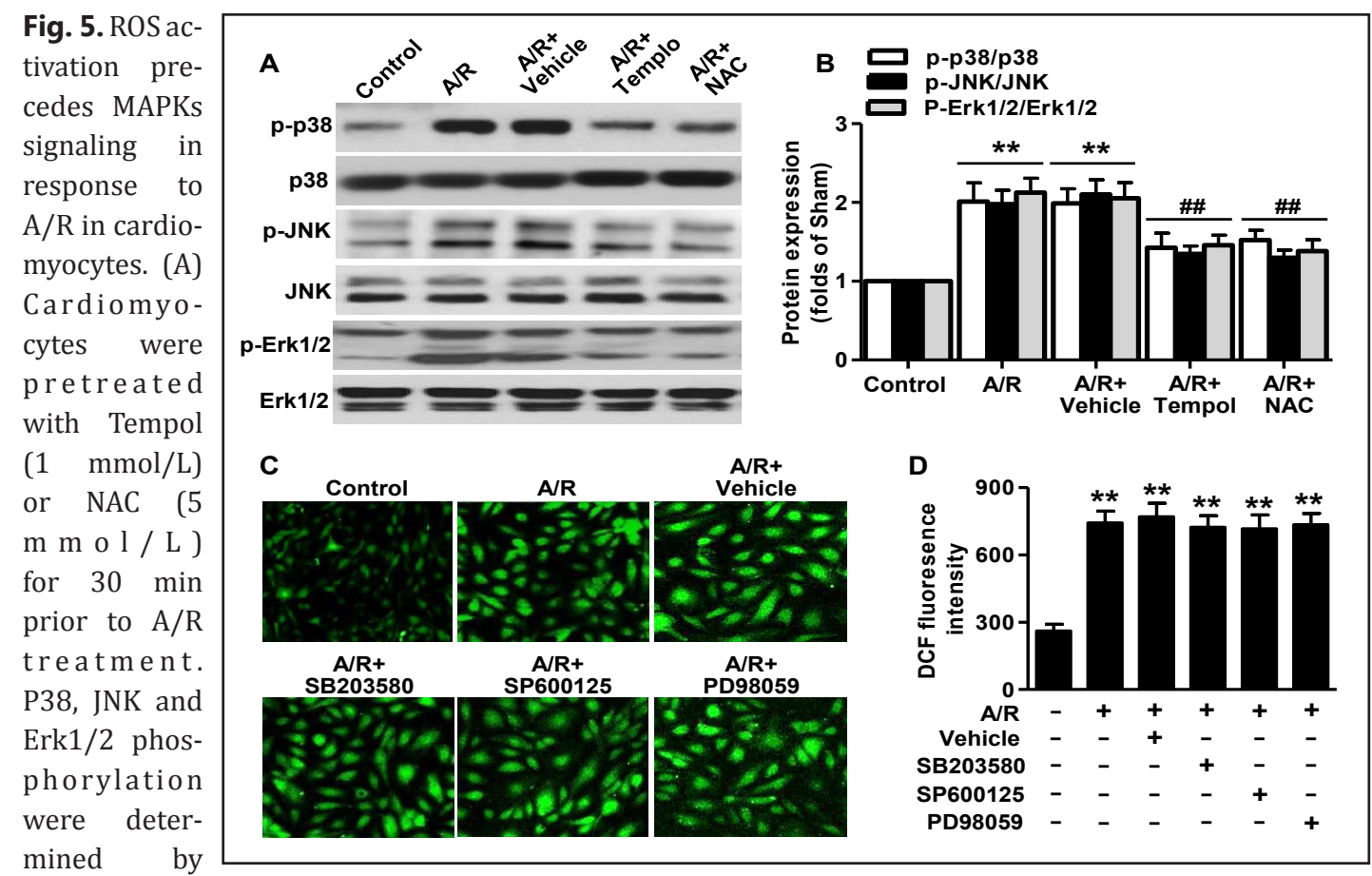

western blot-

ting. (B) Densitometric analysis of the phosphorylated levels of p38, JNK and Erk1/2. (C) The cells were treated with SB203580 $(1 \mu \mathrm{mol} / \mathrm{L})$, SP600125 $(10 \mu \mathrm{mol} / \mathrm{L})$ or PD98059 $(10 \mu \mathrm{mol} / \mathrm{L})$ for $30 \mathrm{~min}$ before A/R treatment. ROS production was measured by $\mathrm{H}_{2}$ DCF-DA $(10 \mu \mathrm{mol} / \mathrm{L})$ staining. (D) Quantitative analysis of DCF fluorescence intensity. ${ }^{* *} \mathrm{P}<0.01$ vs. control; $\# \# \mathrm{P}<0.01$ vs. $\mathrm{A} / \mathrm{R}, \mathrm{n}=7$.

western blotting results showed a significant phosphorylation of p38, JNK and Erk1/2, three MAPKs, upon I/R operation (Fig. 4C and D). To further investigate which one of two signalings was or whether both were responsible for myocardial apoptosis, we employed pharmacological inhibitors of ROS and MAPKs, and then measured their effects on A/Rinduced cardiomyocyte apoptosis in vitro, a condition mimicking in vivo I/R. The Annexin V-FITC/PI data showed that A/R treatment increased the apoptotic rate to $61.4 \pm 7.5 \%$. In contrast to I/R, the apoptotic cell population were markedly decreased by antioxidant (Tempol or NAC), p38 inhibitor (SB203580), JNK inhibitor (SP600125) or Erk1/2 inhibitor (PD98059) alone (Fig. 4E and F). Consistently, these inhibitors also significantly reduced TUNEL-positive cells induced by A/R treatment (Fig. 4G and H). Taken together, these data indicate that I/R-induced cardiomyocyte apoptosis is mediated by ROS and MAPK signaling pathways.

\section{ROS is the upstream of MAPKs in A/R-induced response}

To test whether the increase in ROS production precedes MAPK activation, cardiomyocytes were incubated with antioxidants prior to A/R treatment. Western blotting analysis demonstrated that Tempol or NAC substantially blocked the MAPKs activation as shown by significantly inhibiting A/R-induced p38, JNK and Erk1/2 phosphorylation (Fig. 5A and B). However, SB203580, SP600125 or PD98059 had no effect on A/R-induced the increase in ROS production in cardiomyocyte (Fig. 5C and D). These findings suggest that A/R-mediated ROS activation is the upstream of MAPKs phosphorylation.

Forced TIMP3 expression mitigates cardiomyocyte apoptosis by inhibiting ROS/MAPKS signaling

We further examined whether TIMP3 is involved in I/R-activated ROS/MAPKs pathway, which in turn leads to regulate cardiomyocyte apoptosis. As shown in Fig. 6A, I/R-induced 


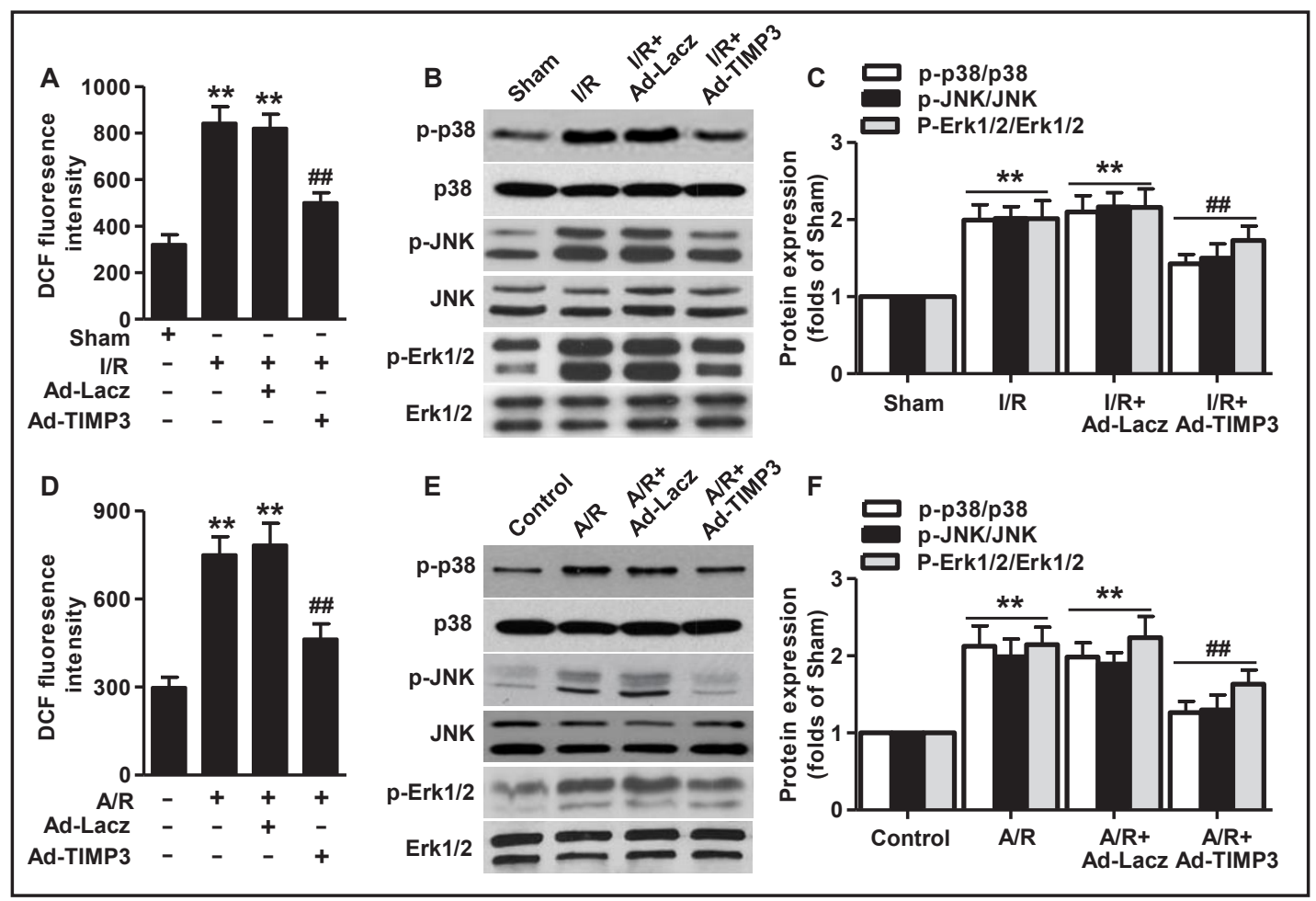

Fig. 6. TIMP3 inhibits ROS/MAPKs pathway activation in response to I/R or A/R. (A) ROS production in heart sections were examined by $\mathrm{H}_{2}$ DCF-DA. Quantitative measurement of DCF fluorescence intensity. (B) Western blotting analysis of p38, JNK and Erk1/2 phosphorylation in heart tissues. (C) Densitometric analysis of the phosphorylated levels of $\mathrm{p} 38$, JNK and Erk1/2. ${ }^{* *} \mathrm{P}<0.01$ vs. sham; $\# \# \mathrm{P}<0.01 \mathrm{vs}$. I/R, $\mathrm{n}=8$. (D) Cardiomyocytes were treated with Ad-Lacz or Ad-TIMP3 for $24 \mathrm{~h}$ before A/R treatment. ROS production was detected and quantitative measurement was performed. (E) P38, JNK and Erk1/2 phosphorylation in cardiomyocytes were determined by western blotting analysis. (F) Densitometric analysis of the phosphorylated levels of p38, JNK and Erk1/2. ${ }^{* *} \mathrm{P}<0.01$ vs. control; $\# \# \mathrm{P}<0.01$ vs. $\mathrm{A} / \mathrm{R}, \mathrm{n}=6$.

ROS production was significantly inhibited by TIMP3 overexpression. Additionally, we observed that the phosphorylation of p38, JNK and Erk1/2 was dramatically reduced in Ad-TIMP3-treated I/R mice (Fig. 6B and C). Moreover, we also confirmed these results by treating cardiomyocytes with A/R condition. Similarly, A/R-triggered ROS production and p38, JNK and Erk1/2 phosphorylation were all alleviated in cells infected Ad-TIMP3, but not Ad-Lacz (Fig. 6D-F). These results demonstrate that the inhibitory effect of TIMP3 on cardiomyocyte apoptosis was mediated, at least in part, by inhibiting ROS/MAPKs activation.

\section{Discussion}

Although the survival of patients with ischemic heart diseases has been improved due to different forms of reperfusion therapy, the prognosis of patients is still grim because of I/R-induced injury [1]. Here, our study shows that TIMP3 upregulation ameliorates I/Rinduced myocardial infarction, fibrosis, dysfunction and heart failure through attenuating myocardial apoptosis. Mechanistically, this is the first report on the anti-apoptotic function of TIMP3 in cardiomyocytes, which we further demonstrates that is mediated, at least in part by inhibiting ROS/MAPKs axis.

TIMP3 expression is closely associated with multiple cardiovascular disorders. It has previously been shown that TIMP3 expression is reduced in atherosclerosis, abdominal aortic aneurysm and type 2 diabetes mellitus from human subjects or experimental 
models [10, 26, 27]. The abnormal TIMP3 expression served as a causal mediator of the corresponding pathophysiologic process $[10,11,13,15]$. Notably, TIMP3 is highly expressed in heart, and its expression is also decreased in myocardial infarction animal model and heart failure patients $[28,29]$. This downregulation was thought to be associated with dilated cardiomyopathy, exacerbated LV dysfunction and increased mortality of heart failure $[3,16-18,28]$. Recent study has provided evidence supporting that re-expression of TIMP3 protects against remodeling-induced myocardial infarction, possibly through reducing MMP activity and promoting coronary angiogenesis [9]. Nevertheless, apart from the above study, there is no information related to the cardiac protective role of TIMP3 in myocardial dysfunction, in particular induced by I/R injury. Here, we tested whether restoration of TIMP3 in myocardium, could ameliorate I/R-induced myocardial injury. We found that upregulation of TIMP3 by adenoviral infection attenuated I/R-induced myocardia infarct size, cytotoxicity, remodeling, contractile dysfunction and mortality. The protective role of TIMP3 was also confirmed in the severe cardiotoxicity induced by Dox in vivo. Our data showed that TIMP3 overexpression was able to reduce infarct size upon Dox treatment, concomitantly with lowered LDH release, fibrosis degree and mortality. These results further demonstrate that TIMP3 is indispensable for myocardial dysfunction.

Previous studies have suggested that I/R injury can cause irreversible myocardial apoptosis and necrosis, the degree of which markedly influences the treatment effects and prognosis of myocardial infarction [4, 5, 22]. Myocardial apoptosis occurs at the onset of I/R injury and almost sustains throughout the whole process of cardiac remodeling and dysfunction, indicating that cardiomyocyte apoptosis is the major pathological indicator of myocardial injury [30,31]. Hence, inhibition of cardiomyocyte apoptosis can ameliorate myocardial dysfunction after I/R injury and limit myocardial remodeling development [32]. Although previous studies have revealed that TIMP3 plays an important role in regulating apoptotic activity in certain cancers $[33,34]$, prior to present study, it is unclear whether TIMP3 is involved in myocardial apoptosis undergoing I/R injury. The present data demonstrated that upregulation of TIMP3 inhibited myocardial apoptosis, as evidenced by reduced TUNEL-positive cells. These results were also supported by the changes of apoptosis-related molecules through western blotting analysis. Bcl-2, Bax, Caspase-3 and Caspase- 9 are known as apoptosis-related proteins, with Bcl-2 being an anti-apoptosis factor, while Bax, Caspase- 3 and Caspase- 9 as pro-apoptotic molecules [2]. We found that the ratio of Bax/Bcl-2, Cleaved Caspase-3 and Cleaved Caspase- 9 expression were all increased after reperfusion, as compared to sham group, while these upregulation was significantly suppressed by TIMP3 overexpression. Therefore, these results suggest that upregulation of TIMP3 alleviates I/R-induced injury via inhibiting myocardial apoptosis.

Oxidative stress-mediated ROS is tightly connected with cardiac dysfunction $[4,32]$. The imbalance between the production and scavenging of ROS can cause an irreversible damage to cells, eventually leading to cell apoptosis [22]. Antioxidant agents have been considered as a potent therapeutic approach for the treatment of I/R injury [23]. MAPKs, including p38, JNK and Erks, are linked to ROS production, which has a pivotal role in cardiovascular diseases. The extent of MAPKs phosphorylation is a critical determinant in deciding cell death and survival, and excessive activation of MAPKs can result in detrimental effects $[24,25]$. In the current study, we found activation of ROS and MAPKs signaling in heart of I/R mice, suggesting the involvement of ROS and MAPKs in cardiac I/R injury. Indeed, our in vivo and in vitro data showed that both ROS and MAPKs mediated I/R- and A/R-induced cardiomyocyte apoptosis, because pharmacological inhibition of ROS or MAPKs almost completely abolished the increase in apoptotic cell population, in line with the important roles of ROS and MAPKs in regulating apoptotic heart cell death [23, 24, 35]. Since ROS and MAPKs seem to be involved in the same signaling pathway, we proceeded to test the sequence of ROS and MAPKs. Our present data showed that inhibition of ROS with antioxidant agents could substantially block MAPKs activation, while MAPKs inhibitors failed to produce significant effect on A/R-induced ROS production. These results indicate that ROS is the upstream of MAPKs in the I/R-induced response. Interestingly, upregulation

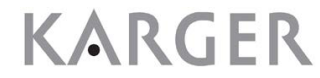


Liu et al.: the Cardiac Protective Effect of TIMP3

of TIMP3 markedly abrogated I/R- or A/R-induced the activation of ROS/MAPKs signaling. The present findings demonstrate that TIMP3 is an upstream regulator of ROS/MAPKs axis, suggesting it may hold great promise as a therapeutic target.

\section{Conclusion}

Our present study demonstrates that TIMP3 protects the myocardium against I/R injury by inhibiting myocardial apoptosis through ROS-initiated MAPKs pathway. These findings suggest that upregulation of TIMP3 expression may provide an efficient approach for the amelioration of myocardial injury induced by I/R.

\section{Acknowledgements}

This work was supported by Key Science and Technology Program of Shanxi Province (No. 2014K11-03-03-04).

\section{Disclosure Statement}

The authors have declared that no competing interests exist.

\section{References}

1 Cannon R0, 3rd: Mechanisms, management and future directions for reperfusion injury after acute myocardial infarction. Nat Clin Pract Cardiovasc Med 2005;2:88-94.

-2 Muzumdar RH, Huffman DM, Calvert JW, Jha S, Weinberg Y, Cui L, Nemkal A, Atzmon G, Klein L, Gundewar S, Ji SY, Lavu M, Predmore BL, Lefer DJ: Acute humanin therapy attenuates myocardial ischemia and reperfusion injury in mice. Arterioscler Thromb Vasc Biol 2010;30:1940-1948.

-3 Tian H, Cimini M, Fedak PW, Altamentova S, Fazel S, Huang ML, Weisel RD, Li RK: TIMP-3 deficiency accelerates cardiac remodeling after myocardial infarction. J Mol Cell Cardiol 2007;43:733-743.

-4 Liu YF, Chu YY, Zhang XZ, Zhang M, Xie FG, Zhou M, Wen HH, Shu AH: TGFbeta1 protects myocardium from apoptosis and oxidative damage after ischemia reperfusion. Eur Rev Med Pharmacol Sci 2017;21:15511558.

5 Wang X, Cao Y, Shen M, Wang B, Zhang W, Liu Y, He X, Wang L, Xia Y, Ding M, Xu X, Ren J: DIDS reduces ischemia/reperfusion-induced myocardial injury in rats. Cell Physiol Biochem 2015;35:676-688.

6 Zile MR, Baicu CF, Gaasch WH: Diastolic heart failure--abnormalities in active relaxation and passive stiffness of the left ventricle. N Engl J Med 2004;350:1953-1959.

7 Greenlee KJ, Werb Z, Kheradmand F: Matrix metalloproteinases in lung: multiple, multifarious, and multifaceted. Physiol Rev 2007;87:69-98.

8 Gill SE, Parks WC: Metalloproteinases and their inhibitors: regulators of wound healing. Int J Biochem Cell Biol 2008;40:1334-1347.

9 Takawale A, Zhang P, Azad A, Wang W, Wang X, Murray AG, Kassiri Z: Myocardial overexpression of TIMP3 following myocardial infarction exerts beneficial effects through promoting angiogenesis and suppressing early proteolysis. Am J Physiol Heart Circ Physiol 2017;313:H224-H236.

10 Di Gregoli K, Mohamad Anuar NN, Bianco R, White SJ, Newby AC, George SJ, Johnson JL: MicroRNA-181b Controls Atherosclerosis and Aneurysms Through Regulation of TIMP-3 and Elastin. Circ Res 2017;120:4965.

11 Arpino V, Mehta S, Wang L, Bird R, Rohan M, Pape C, Gill SE: Tissue inhibitor of metalloproteinases 3-dependent microvascular endothelial cell barrier function is disrupted under septic conditions. Am J Physiol Heart Circ Physiol 2016;310:H1455-1467. 


\section{Cellular Physiology Cell Physiol Biochem 2017;44:1011-1023 \begin{tabular}{l|l|l}
\hline and Biochemistry 10.1159/000485401 & $\begin{array}{l}\text { C } 2017 \text { The Author(s). Published by S. Karger AG, Basel } \\
\text { www.karger.com/cpb }\end{array}$ \\
\hline
\end{tabular}}

Liu et al.: the Cardiac Protective Effect of TIMP3

12 Basu R, Lee J, Morton JS, Takawale A, Fan D, Kandalam V, Wang X, Davidge ST, Kassiri Z: TIMP3 is the primary TIMP to regulate agonist-induced vascular remodelling and hypertension. Cardiovasc Res 2013;98:360-371.

13 Stohr R, Cavalera M, Menini S, Mavilio M, Casagrande V, Rossi C, Urbani A, Cardellini M, Pugliese G, Menghini R, Federici M: Loss of TIMP3 exacerbates atherosclerosis in ApoE null mice. Atherosclerosis 2014;235:438-443.

14 Casagrande V, Menghini R, Menini S, Marino A, Marchetti V, Cavalera M, Fabrizi M, Hribal ML, Pugliese G, Gentileschi P, Schillaci O, Porzio O, Lauro D, Sbraccia P, Lauro R, Federici M: Overexpression of tissue inhibitor of metalloproteinase 3 in macrophages reduces atherosclerosis in low-density lipoprotein receptor knockout mice. Arterioscler Thromb Vasc Biol 2012;32:74-81.

15 Qi JH, Ebrahem Q, Moore N, Murphy G, Claesson-Welsh L, Bond M, Baker A, Anand-Apte B: A novel function for tissue inhibitor of metalloproteinases-3 (TIMP3): inhibition of angiogenesis by blockage of VEGF binding to VEGF receptor-2 Nat Med 2003;9:407-415

16 Kassiri Z, Defamie V, Hariri M, Oudit GY, Anthwal S, Dawood F, Liu P, Khokha R: Simultaneous transforming growth factor beta-tumor necrosis factor activation and cross-talk cause aberrant remodeling response and myocardial fibrosis in Timp3-deficient heart. J Biol Chem 2009;284:29893-29904.

17 Stawowy P, Margeta C, Kallisch H, Seidah NG, Chretien M, Fleck E, Graf K: Regulation of matrix metalloproteinase MT1-MMP/MMP-2 in cardiac fibroblasts by TGF-beta1 involves furin-convertase. Cardiovasc Res 2004;63:87-97.

18 Kassiri Z, Oudit GY, Sanchez O, Dawood F, Mohammed FF, Nuttall RK, Edwards DR, Liu PP, Backx PH, Khokha R: Combination of tumor necrosis factor-alpha ablation and matrix metalloproteinase inhibition prevents heart failure after pressure overload in tissue inhibitor of metalloproteinase-3 knock-out mice. Circ Res 2005;97:380-390.

19 Tong G, Zhang B, Zhou X, Zhao J, Sun Z, Tao Y, Pei J, Zhang W: Kappa-Opioid Agonist U50, 488H-Mediated Protection Against Heart Failure Following Myocardial Ischemia/Reperfusion: Dual Roles of Heme Oxygenase-1 Cell Physiol Biochem 2016;39:2158-2172.

20 Yang J, Guo X, Yang J, Ding JW, Li S, Yang R, Fan ZX, Yang CJ: RP105 Protects Against Apoptosis in Ischemia/ Reperfusion-Induced Myocardial Damage in Rats by Suppressing TLR4-Mediated Signaling Pathways. Cell Physiol Biochem 2015;36:2137-2148.

-21 Peng W, Zhang Y, Zheng M, Cheng H, Zhu W, Cao CM, Xiao RP: Cardioprotection by CaMKII-deltaB is mediated by phosphorylation of heat shock factor 1 and subsequent expression of inducible heat shock protein 70 Circ Res 2010;106:102-110.

22 Zhang T, Zhang Y, Cui M, Jin L, Wang Y, Lv F, Liu Y, Zheng W, Shang H, Zhang J, Zhang M, Wu H, Guo J, Zhang $\mathrm{X}, \mathrm{Hu}$ X, Cao CM, Xiao RP: CaMKII is a RIP3 substrate mediating ischemia- and oxidative stress-induced myocardial necroptosis. Nat Med 2016;22:175-182.

23 Wu SZ, Tao LY, Wang JN, Xu ZQ, Wang J, Xue YJ, Huang KY, Lin JF, Li L, Ji KT: Amifostine Pretreatment Attenuates Myocardial Ischemia/Reperfusion Injury by Inhibiting Apoptosis and Oxidative Stress. Oxid Med Cell Longev 2017;2017:4130824.

-24 Rose BA, Force T, Wang Y: Mitogen-activated protein kinase signaling in the heart: angels versus demons in a heart-breaking tale. Physiol Rev 2010;90:1507-1546.

25 Jeffrey KL, Camps M, Rommel C, Mackay CR: Targeting dual-specificity phosphatases: manipulating MAP kinase signalling and immune responses. Nat Rev Drug Discov 2007;6:391-403.

26 Cardellini M, Menghini R, Martelli E, Casagrande V, Marino A, Rizza S, Porzio O, Mauriello A, Solini A, Ippoliti A, Lauro R, Folli F, Federici M: TIMP3 is reduced in atherosclerotic plaques from subjects with type 2 diabetes and increased by SirT1 Diabetes 2009;58:2396-2401.

27 Monroy A, Kamath S, Chavez AO, Centonze VE, Veerasamy M, Barrentine A, Wewer JJ, Coletta DK, Jenkinson C, Jhingan RM, Smokler D, Reyna S, Musi N, Khokka R, Federici M, Tripathy D, DeFronzo RA, Folli F: Impaired regulation of the TNF-alpha converting enzyme/tissue inhibitor of metalloproteinase 3 proteolytic system in skeletal muscle of obese type 2 diabetic patients: a new mechanism of insulin resistance in humans. Diabetologia 2009;52:2169-2181.

28 Kandalam V, Basu R, Abraham T, Wang X, Awad A, Wang W, Lopaschuk GD, Maeda N, Oudit GY, Kassiri Z: Early activation of matrix metalloproteinases underlies the exacerbated systolic and diastolic dysfunction in mice lacking TIMP3 following myocardial infarction. Am J Physiol Heart Circ Physiol 2010;299:H10121023. 


\section{Cellular Physiology Cell Physiol Biochem 2017;44:1011-1023 \begin{tabular}{l|l|l} 
DOI: 10.1159/000485401 & $\begin{array}{l}\text { O 2017 The Author(s). Published by S. Karger AG, Basel } \\
\text { www.karger.com/cpb }\end{array}$ \\
\hline
\end{tabular}}

29 Li YY, Feldman AM, Sun Y, McTiernan CF: Differential expression of tissue inhibitors of metalloproteinases in the failing human heart. Circulation 1998;98:1728-1734.

-30 Zhu J, Yao K, Wang Q, Guo J, Shi H, Ma L, Liu H, Gao W, Zou Y, Ge J: Ischemic Postconditioning-Regulated miR-499 Protects the Rat Heart Against Ischemia/Reperfusion Injury by Inhibiting Apoptosis through PDCD4. Cell Physiol Biochem 2016;39:2364-2380.

-31 Zhang JY, Wu F, Gu XM, Jin ZX, Kong LH, Zhang Y, Zhou JJ, Gao F: The Blockade of Transmembrane Cl(-) Flux Mitigates I/R-Induced Heart Injury via the Inhibition of Calpain Activity. Cell Physiol Biochem 2015;35:2121-2134.

-32 Sun Y, Zhao D, Yang Y, Gao C, Zhang X, Ma Z, Jiang S, Zhao L, Chen W, Ren K, Yi W, Gao F: Adiponectin exerts cardioprotection against ischemia/reperfusion injury partially via calreticulin mediated anti-apoptotic and anti-oxidative actions. Apoptosis 2017;22:108-117.

33 Shen B, Jiang Y, Chen YR, Zheng HC, Zeng W, Li YY, Yin A, Nie Y: Expression and inhibitory role of TIMP-3 in hepatocellular carcinoma. Oncol Rep 2016;36:494-502.

34 Kubatka P, Uramova S, Kello M, Kajo K, Kruzliak P, Mojzis J, Vybohova D, Adamkov M, Jasek K, Lasabova Z, Zubor P, Fialova S, Dokupilova S, Solar P, Pec M, Adamicova K, Danko J, Adamek M, Busselberg D: Antineoplastic effects of clove buds (Syzygium aromaticum L.) in the model of breast carcinoma. J Cell Mol Med 2017;10.1111/jcmm.13197

35 Dougherty JA, Kilbane Myers J, Khan M, Angelos MG, Chen CA: Dual-Specificity Phosphatase 4 Overexpression in Cells Prevents Hypoxia/Reoxygenation-Induced Apoptosis via the Upregulation of eNOS. Front Cardiovasc Med 2017;4:22. 